Published in final edited form as:

Nano Lett. 2019 August 14; 19(8): 5762-5768. doi:10.1021/acs.nanolett.9b02402.

\title{
Measuring Nanoparticle Polarizability using Fluorescence Microscopy
}

\author{
Wenhan Cao ${ }^{1}$, Margaret Chern'르. Allison M. Dennis ${ }^{2,3}$, Keith A. Brown ${ }^{1,2,4,{ }^{*}}$ \\ ${ }^{1}$ Department of Mechanical Engineering, Boston University, Boston, MA, 02215, USA \\ 2Division of Materials Science \& Engineering, Boston University, Boston, MA, 02215, USA \\ ${ }^{3}$ Department of Biomedical Engineering, Boston University, Boston, MA, 02215, USA \\ ${ }^{4}$ Physics Department, Boston University, Boston, MA, 02215, USA
}

\begin{abstract}
Using a novel method developed to quantify the polarizability of photoluminescent nanoparticles in water, we present experimental observation of the extraordinary polarizability exhibited by nanoparticles of commensurate size to the Debye screening length, confirming previously reported theory. Semiconductor quantum dots (QDs) are ideal model nanoparticles to demonstrate this assay, due to their tunable size and bright photoluminescence. This assay is based upon microfluidic chambers with microelectrodes that generate trapping potentials that are weaker than thermal energy. By comparing the local electric field strength and variations in QD concentration, their polarizability was computed and found to agree with estimates based upon the hydrodynamic diameter found using light scattering. Strikingly, the polarizability of the nanoparticles increased 30-fold in low salt conditions compared to high salt conditions due to the increased thickness of the Debye layer relative to the particle radius. In addition to providing evidence that corroborates theoretical work studying direct solutions to the Poisson-Nernst-Planck equations, these observations provide an explanation for previously observed conductivity dependence of biomolecule polarizability. As the polarizability of nanoparticles is of high importance to the electrically-directed assembly of particles, as well as their interactions with other materials in complex environments, we anticipate that these results will be highly relevant to ongoing efforts in materials by design and nanomedicine.
\end{abstract}

\section{Graphical Abstract}

\footnotetext{
*Corresponding Author Keith A. Brown, brownka@bu.edu. Author Contributions

The manuscript was written through contributions of all authors. All authors have given approval to the final version of the manuscript. Supporting Information.

Experimental details for microfluidic chamber fabrication, quantum dot synthesis, fluorescence assay, and lumped element model of electrodes. SI Figures include a scheme depicting the fluid cell fabrication process, measurements of chamber impedance, measurements of quantum dot fluorescence intensity vs. concentration, time-dependent measurements of fluorescence showing the stability of the concentration enhancement, a schematic of the region used for finite element analysis, and a negative stained TEM image of the QDs. This material is available free of charge via the Internet at http://pubs.acs.org.
} 

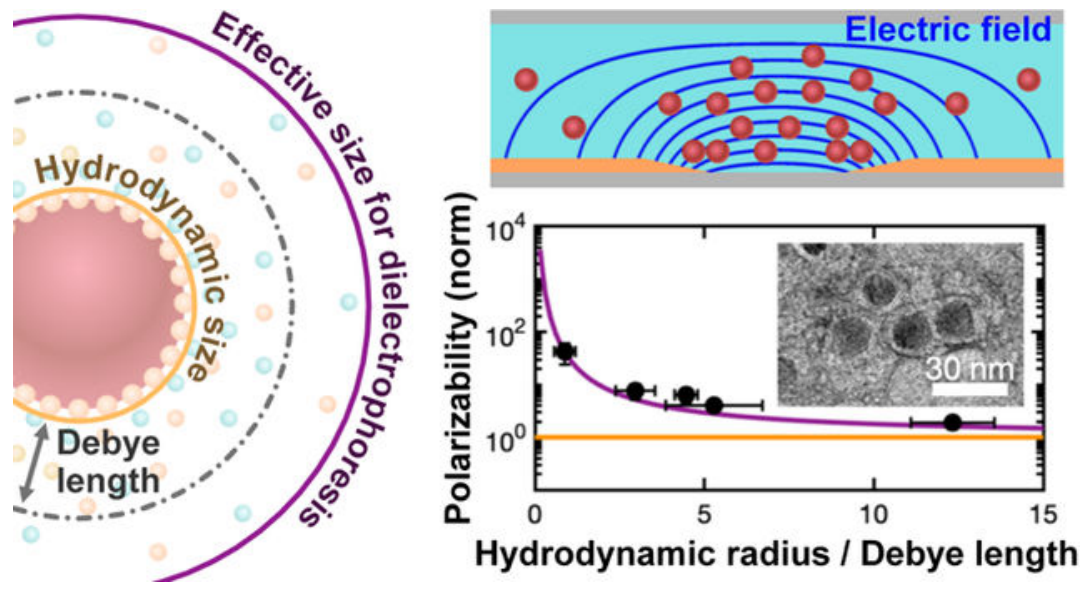

\section{Keywords}

Nanoparticle; Polarizability; Dielectrophoresis; Field-directed assembly; Quantum Dot; Electrostatic Double Layer

Electric fields represent a versatile and dynamic tool for interrogating and manipulating nanomaterials and biomaterials in solution. ${ }^{1}$ Two primary ways that electric fields can directly exert forces on materials are electrophoresis, or the motion of charged materials along electric field lines, and dielectrophoresis (DEP), which is the motion of polarizable objects along gradients of field intensity. ${ }^{2}$ DEP, in particular, has become a widely used tool for separating, sorting, trapping, and manipulating objects in solution including cells, microparticles, nanoparticles, and biomolecules. ${ }^{1,3-5}$ Just as one needs to know an object's zeta potential in order to predict its electrophoretic behavior, accurately predicting the effect of DEP requires measuring the polarizability of the material, which is a frequencydependent property that depends on the composition of the particle and medium. While a number of techniques have been developed to measure polarizability, those that have been used with particles are largely limited to the study of microscopic objects that can be individually optically resolved. ${ }^{6-15}$ Despite the growing importance of nanoparticles ranging from fields including nanomedicine and materials assembly, ${ }^{16}$ there are very few techniques that are capable of quantifying the polarizability of nanoparticles, and values for the polarizability of nanoparticles in solution have not been reported. ${ }^{17-20}$ This is especially unfortunate because theory predicts that the polarizability of nanoscale objects could change substantially as the size of the particle approaches the Debye screening length of the electrolytic fluid (Figure 1A). ${ }^{21-28}$ The closest experimental validation of these theories has come from impedance spectroscopy measurements in which the impedance of dense suspensions ( $>1 \%$ volume fraction) of particles are studied vs. frequency. ${ }^{29-31}$ Despite focusing on relatively large particles (typically $>150 \mathrm{~nm}$ ), these studies can access regimes where the Debye length approaches the particle radius, however connection to the individual particle polarizability is limited by operation at high volume fractions. As a further example of the need to understand how free charge affects field-induced particle motion, recent measurements and theoretical predictions of electrophoretic motion of nanoscale objects have shown that nanoscale objects behave differently with enhanced mobility emerging 
when the particle becomes commensurate in size with the ionic screening length. ${ }^{28,} 32$ While studies of particles are limited, measurements of the polarizability of biomolecules have proceeded over the past decades and have resulted in approaches that could potentially provide measurements of polarizability of abiotic nanoscale objects. ${ }^{33-45}$ However, measurements of DNA and other biomolecules have revealed puzzling behavior regarding the interaction between salt concentration and polarizability that has not yet been reconciled, likely due to the difficulty in accurately modeling biomolecules as simple structures. $42,44,46,47$

Here, we develop a method to measure the polarizability of photoluminescent nanoparticles and use it to quantify the polarizability of semiconductor quantum dots (QDs), which is found to dramatically increase at low salt concentrations (Figure 1B). The polarizability assay relies upon the bright photoluminescence inherent to QDs that allows their local concentration to be measured using fluorescence microscopy. Specifically, we generate microfluidic chambers and apply modest electric fields that give rise to DEP potentials with magnitudes approaching thermal energy, allowing the local relative concentration to equilibrate (Figure 1C). By quantifying the change in local concentration, polarizability is computed and compared to the hydrodynamic size found using light scattering. At high salt concentrations, where the particle is substantially larger than the Debye screening length, we find that standard models based upon interfacial polarization match values from experiment. More interestingly, at lower salt concentrations, in which the Debye screening length becomes comparable to the particle size, QDs are found to exhibit a polarizability that is nearly 30 times larger than what is predicted by simple models, validating the need to include space charge effects from the Debye screening layer in the polarizability of the particles. In addition to providing experimental evidence that can be used to validate theories that apply to the polarizability of nanoscale objects, these results suggest that DEP has a larger impact on nanoparticle behavior in low conductivity electrolytic solutions than would be predicted by simple assumptions. Since this approach is valid for particles that are too small to be individually resolved, it can be used to quantify the dielectric properties of fluorescently-tagged biomolecules and other nanoparticles.

Fundamentally, DEP is the movement of an object due to the force acting its the induced dipole in a non-uniform electric field $\vec{E}$. In particular, an isotropic polarizable particle can be described by a polarizability $a$ that defines $\vec{p}=\alpha \vec{E}$, where $\vec{p}$ is the induced dipole moment of the particle. ${ }^{2}$ In the electrostatic limit, the energy $U_{D E P}$ associated with the interaction between $\vec{p}$ and $\vec{E}$ is,

$$
U_{D E P}=-\frac{1}{2} \alpha E^{2}
$$

Studies on the DEP behavior of micron-scale objects such as microparticles and biological cells are usually performed when $\left|U_{D E P}\right| \gg k_{B} T$, with Boltzmann constant $k_{B}$ and absolute temperature $T$, indicating that DEP is substantially stronger than Brownian motion. 6-10, 13, 14, 48 This, together with individual particles being optically resolvable, enables indirect quantification of a through measurements of velocity resulting from a competition between drag and DEP, $6,11,13,15$ the external flow speed needed to dislodge a particle from 
a stationary DEP trap, ${ }^{7,} 39$ or the trajectory of particles through curving fluid flows. ${ }^{41}$ In interpreting such measurements, material properties are typically estimated using analytical models such as the expression for a spherical particle,

$$
\alpha=4 \pi a^{3} \varepsilon_{m} \operatorname{Re}\{K\},
$$

where $K$ is known as the Clausius-Mossotti or dipole factor given by, ${ }^{2}$

$$
K=\frac{\widetilde{\varepsilon}_{p}-\widetilde{\varepsilon}_{m}}{\widetilde{\varepsilon}_{p}+2 \widetilde{\varepsilon}_{m}}
$$

where $\widetilde{\varepsilon}_{m}$ is the complex permittivity of the medium, $\widetilde{\varepsilon}_{p}$ is the complex permittivity of the particle, and $a$ is the radius of the particle. Since water has a large real dielectric permittivity $\left(\varepsilon_{m}=78 \varepsilon_{0}\right.$, with permittivity of free space $\varepsilon_{0}$ ), it is often the case for dielectric particles that $\varepsilon_{p}<\varepsilon_{m}$, which leads to $a<0$ in the high frequency limit and indicates that particles will be pushed from regions of high field intensity to those with low field intensity in a phenomenon known as negative DEP (nDEP). The situation becomes more intricate when the solution is taken to have a finite real medium conductivity $\sigma_{M}$ as this contributes to $\widetilde{\varepsilon}_{m}{ }^{2}$ Further,

Maxwell-Wagner (MW) interfacial polarization predicts that the presence of ions in the medium that accumulate around charged particles can be modeled as surface conductivity that increases $\left|\widetilde{\varepsilon}_{p}\right|$ at low frequencies such that $\left|\widetilde{\varepsilon}_{p}\right|>\left|\widetilde{\varepsilon}_{m}\right|$, resulting in $a>0$ and particles moving to regions of high field intensity in an effect known as positive DEP (pDEP). Modeling the ions screening the particle as a conductive layer of infinitesimal thickness is typically acceptable for micron-scale particles as the Debye length $\lambda_{D} \ll a^{49}$

In contrast with studies of microscopic objects in which individual particles can be optically resolved, there is a rich tradition of studying the dielectric response of biomolecules such as DNA using techniques that do not require resolving individual particles. Early studies included those that utilized electrically-induced birefringence of DNA solutions, ${ }^{34}$ fluorescence anisotropy induced by electric fields, ${ }^{35}$ or impedance spectroscopy to measure the dielectric properties of biomolecular solutions. ${ }^{36,43}$ While these studies have produced an impressive body of knowledge, including how DNA polarizability scales with the number of base pairs when in different configurations, ${ }^{42}$ there have been puzzling results that point to deeper questions about the interaction between salt and the biomolecules. ${ }^{46}$ For instance, high conductivity buffers have been observed to result in less effective trapping than low conductivity buffers in both nanogap electrodes and among insulating structures that locally concentrate fields along a channel. ${ }^{42,47}$ The same trend (increasing conductivity results in reduced trapping) has been observed when studying the behavior of proteins such as IgG. ${ }^{44}$ As biomolecules are much smaller than the microparticles that have been extensively studied in a DEP context, it is likely that their size is such that $\lambda_{D^{\sim}}$, which means that the Debye layer can no longer be modeled as a conductive shell (which would indicate that $|K| \leq 1$ ), but rather the full Poisson-Nernst-Planck (PNP) equations must be solved to predict $a$, allowing the possibility of $K>1$, or an apparent increase in the particle size. ${ }^{49}$ Despite the presence of this fascinating transition, it has not been systematically studied as it requires monodisperse photoluminescent nanoparticles coupled with a method for the ensemble measurement of $a$ as the nanoscopic particles cannot be independently resolved. 
We developed a system to measure the $a$ of model nanoparticles: thick-shelled CdSe/CdS QDs. ${ }^{50-54}$ QDs represent an ideal testbed for this process, as they are highly monodisperse and exhibit extremely bright photoluminescence. ${ }^{55}$ As any fluorophore, QDs exhibit a linear relationship between concentration and photoluminescent intensity, as long as the fluorophore is dilute enough to avoid inner filter effects and other forms of self-quenching. ${ }^{56}$ This correlation is easily observed in a spectrophotometer, but quantitatively converting fluorescence to local concentration in epifluorescence microscopy images is challenging, as out of focus light may lead to a large background. We hypothesized that physically confining the QDs to a volume with a vertical height on the order of the focal depth could allow for more quantitative evaluation. To generate uniform electric fields inside such a microfluidic chamber, we microfabricated an array of co-planar strip electrodes composed of transparent indium tin oxide (ITO) such that the $5 \mu \mathrm{m}$ height of the chamber is smaller than the $15 \mu \mathrm{m}$ spacing between electrodes (Figure S2, Figure 2A). By measuring the topographic profile of these electrodes (Figure 2B), it is possible to compute the precise electric field vs. height $z$ in the chamber (Figure 2C). Importantly, in the middle of the gap region between electrodes, the field is computed to be large and nearly constant, while the field in the region above the middle of a given electrode is zero. Thus, these two regions provide a spatially separate but proximal comparison of behavior in a high- and low-field environment.

Before this $a$ measurement scheme can be applied, it is critical to quantify $|\vec{E}|$ inside the gap region. While simulation can connect the applied voltage $V$ with $\vec{E}$, it assumes that the voltage on the electrodes $V_{m}$ matches $V$ and electrode polarization does not reduce the magnitude of the potential experienced by the fluid. In order to determine the window in frequency $f$ in which this is true, we constructed a lumped element circuit model of this system (Figure 2D) that includes the series resistance of the leads, electrode polarization, and the conductive/dielectric behavior of the medium. In particular, the lumped element circuit model includes contributions from the electrode resistance $R_{\mathcal{e}}$, which is estimated to be $450 \Omega$ based on the geometry and sheet resistance of the electrodes, and the medium capacitance $C_{m}$, which is estimated to be $75 \mathrm{pF}$ using a finite element simulation of the electrode geometry. The electrode-solution capacitance $C_{e}$ and medium resistance $R_{m}$ both depend on $\sigma_{m}$ and are found using a parallel plate approximation of electrode polarization and finite element analysis of the electrode geometry, respectively. Details of how these terms are calculated, along with a table of their values, are given in the Supporting Information. Values estimated for these lumped element properties are consistent with impedance spectroscopy of the microfluidic chamber (Supporting Information, Figure S4). This analysis shows that at low frequencies, the voltage is attenuated due to electrode polarization, while at high frequencies, the voltage is attenuated due to series resistance of the electrodes. Using this model, we calculated the range in $f$ in which the voltage on the electrodes is attenuated by less than $1 \mathrm{~dB}$ (Figure 2E). Electrochemical reactions, such as electrolysis, are commonly avoided through operation at high frequency as they require $\sim 1 \mathrm{~V}$ potential across the double layer. ${ }^{2}$ The lumped element model indicates that the voltage across the double layer at $1 \mathrm{MHz}$ is several orders of magnitude smaller than the $\sim 3 \mathrm{~V}$ applied voltages, indicating that electrochemistry should not play a role, consistent with our observations. 
Having fabricated a microfluidic system with a known distribution of $\vec{E}$, we used it to measure the dielectrophoretic activity of QDs. Before applying a voltage, fluorescence images of the chamber filled with a $25 \mathrm{nM}$ solution of poly(maleic anhydride-alt-1octadecene) (PMAO)-coated CdSe/CdS QDs in $12.5 \mathrm{mM}$ borate buffer, $\mathrm{pH} 8.5$, revealed a featureless field (Figure 3A). Upon the application of a sinusoidal voltage at $f=1 \mathrm{MHz}$ with root-mean-squared magnitude $V=0.71 \mathrm{~V}$, light and dark regions emerged above the gap and electrode regions, respectively (Figure 3B). Increasing $V$ to $1.76 \mathrm{~V}$ (Figures 3C) and $2.82 \mathrm{~V}$ (Figures 3D), made the contrast of these striped regions increasingly more distinct. Using image processing, regions of high intensity $I_{H}$ and low intensity $I_{L}$ were identified (Figure $3 \mathrm{E})$, allowing their distributions within a given image to be quantified. Examining how $I_{H}$ and $I_{L}$ change with $V$, it is clear that they are governed by a non-linear relationship with bright regions becoming increasingly bright relative to the dim regions (Figure $3 \mathrm{~F}$ ). To calibrate the relationship between QD concentration and intensity, we observed the fluorescence intensity of different QD concentrations, confirming that, in this microfluidic system and consistent imaging conditions, fluorescence intensity was proportional to QD concentration in the range $0-100 \mathrm{nM}$ (Figure S5). The linearity between concentration and intensity further suggests that QD aggregation is not occurring as this would reduce the fluorescent intensity in a concentration-dependent manner. In addition to maintaining the same imaging and illumination conditions, we account for camera noise in the absence of illumination (dark current) as a constant background subtracted from all images taken with the same gain/exposure settings. It is important to note that microscopy averages the photoluminescence intensity in a hyperboloidal point spread function (denoted by red dashed lines in Figure 2C) while the electric field $E(x, y, z)$ in the fluid chamber varies spatially. Thus, in order to connect the local illumination intensity to a specific $E$, it is important that the $E$ is constant in this region, as is the case in the field maximum between electrodes and field zero above each electrode (further details in Supporting Information).

In the analysis of the electric-field induced concentration shifts, it is critical to note that even at the maximum $V$ considered, $I$ changes by $<30 \%$, which indicates that the particles are not strongly trapped. Specifically, since concentration is governed by a Boltzmann distribution, a $30 \%$ increase in concentration over the background indicates that the trapping energy given by Equation (1) is approximately four times smaller than thermal energy $k_{B} T$, implying that particles are weakly trapped compared with thermal energy. Indeed, a key insight from experiments studying the DEP behavior of DNA is that this low field limit in which $\mid U_{D E P} \lesssim$ $k_{B} T$ features the interplay between Brownian motion and DEP in a manner that can provide information through measurements of escape times or observations of concentration enhancement. ${ }^{33,37,38}$ In this limit, $E$ locally modifies $C$ according to a Boltzmann distribution,

$$
C=\frac{1}{Z} e^{\frac{\alpha E^{2}}{2 k_{B} T}}
$$

where $Z$ is a normalizing factor known as the canonical partition function. Equation (4) has the useful implication that measurements of $C$ can provide a measurement of $a$. Practically, however, there are complications associated with applying this framework, namely that $Z$ is 
changing as DEP concentrates particles in the high field regions and depletes low field regions, so absolute changes in $C$ cannot be trivially connected to the local field strength. We overcome this challenge by analyzing the concentration in the high field regions $C_{H} \propto I_{H^{-}}$ $I_{D a r k}$ relative to the concentration in the low-field regions $C_{L} \propto I_{L}-I_{D a r k}$ to remove the effect of changing $Z$. As the regions directly above the electrodes have no field due to the symmetry of the system, they allow us to directly capture and account for this variation of $Z$ (Supporting Information). Subsequently, these experimental data were used to obtain experimental values of $a$ by fitting to,

$$
\frac{C_{H}}{C_{L}}=b e_{2 k_{B} T d_{e}^{2}}
$$

where $b$ and $a$ are fitting parameters. The effective electrode spacing $d_{e}=15.5 \pm 0.2 \mu \mathrm{m}$ was found using finite element simulation (Supporting Information). Thus, by plotting $\left(I_{H^{-}}\right.$ $\left.I_{\text {Dark }}\right) /\left(I_{L}-I_{\text {Dark }}=C_{H} / C_{L}\right.$ vs. $V$ (Figure 3G), we may fit Equation (5) to compute $a$. In particular, for PMAO coated CdSe/CdS QDs at $\sigma_{m}=303 \mu \mathrm{S} / \mathrm{cm}$, we find $a=(3.8 \pm 0.6) \times$ $10^{-32} \mathrm{C} \cdot \mathrm{m}^{2} / \mathrm{V}$. As this measurement requires that the distribution of QDs is in local equilibrium, we confirmed that the measurement does not vary in time (Figure S6). This experimental control confirms that we are assessing an equilibrium property of the system rather than a kinetic one. As is common in the study of colloids subjected to AC fields, DC effects will not be important as they will time average to zero. ${ }^{2}$ In particular, assuming taking $2 \times 10^{-8} \mathrm{~m}^{2} \mathrm{~V}^{-1} \mathrm{~s}^{-1}$ to be a typical value for the electrophoretic mobility of QDs, ${ }^{57}$ electrophoresis will move the QDs only $\sim 1 \mathrm{~nm}$ during each half cycle of the AC field. The gravitational length for QDs is $\sim 20 \mathrm{~mm}$. Given that the chamber is $5 \mu \mathrm{m}$ tall, gravity will have no effect here.

To explore the relationship between $a$ and QD size, we used Equation (2) to calculate the QD size assuming that $K=1$ and found $a_{D E P}=10.6 \pm 1.7 \mathrm{~nm}$. As a comparison, we utilized dynamic light scattering (DLS) to compute $a_{D L S}=8.5 \pm 0.8 \mathrm{~nm}$, which is consistent with the DEP value. Transmission electron microscopy (TEM) further validated these results by revealing that the inorganic QD cores were $5.9 \pm 0.8 \mathrm{~nm}$ in radius and $9.6 \pm 0.9 \mathrm{~nm}$ with the PMAO coating (Figure 4A, Figure S1). These observations, namely that the apparent size measured by DEP matches the physical size of the particle, are reasonable given that the relatively high salt concentration of this experiment will result in $\lambda_{D}=0.7 \mathrm{~nm}$ (Supporting Information), which is substantially smaller than $a$, indicating that MW theory can be reasonably applied.

While measurements at high salt concentration revealed an agreement between DLS- and DEP-measured size, at lower salt concentration, deviation was observed (Figure 4B). In particular, DEP size measurements of a $25 \mathrm{nM}$ solution of QDs imaged between $V=0.35$ and $2.82 \mathrm{~V}$ in borate buffers diluted with DI water were used to compute $a$ and converted to measurements of $a_{D E P}$ using Equation (2), assuming pDEP $(K=1)$. Strikingly, at $\sigma_{m}<100$ $\mu \mathrm{S} / \mathrm{cm}$, the apparent size of the QDs increased, an effect which is reminiscent of prior observations in biomolecular systems in which lower salt concentrations produced a larger DEP effect. In contrast with the increasing size observed by DEP, the size measured by DLS slightly decreases with decreasing $\sigma_{m}$. This statistically significant decrease could be the 
result of swelling of the amphiphilic polymer coating in high ionic strength solutions, as has been seen in related polymer systems. ${ }^{58}$ The variation in DLS-measured size is unlikely to be related to aggregation as the size of the particles is in all cases consistent with the range measured by TEM, however this warrants further study due to the high variance associated with DLS. While the changing hydrodynamic radius measured by DLS with salt concentration evokes effects such as electroviscosity, this effect tends to make particles appear larger at lower salt concentrations. ${ }^{28,59}$ It is worth noting that local changes in viscosity would not affect the measurement of $a$, as this is purely based upon thermodynamic properties rather than kinetics.

Taking inspiration from theory work in which $a$ is predicted to depend upon the ratio $a / \lambda_{D}$, we plot $a$, as determined by fitting to Equation (5), vs. $a / \lambda_{D}$ (Figure 4C). At each $\sigma_{m}$, we normalize $a$ by $a_{0}$, which is the polarizability computed using Equation (2) assuming that the particle is a perfectly polarizable $(K=1)$ sphere with radius defined by its DLSdetermined hydrodynamic radius $(8.5 \mathrm{~nm})$. Interestingly, $a / a_{0}$ was found to monotonically increase as $a / \lambda_{D}$ decreased, illustrating that increasing the size of the Debye layer makes the particle appear larger at lower salt concentrations. One way to conceptualize the increase in $a$ with decreasing $\sigma_{m}$ is to consider that when $\lambda_{D} \gtrsim a$, the particle will effectively appear larger and will be defined by an effective radius $a_{e f f}=a+\beta \lambda_{D}$, where $\beta$ is a dimensionless factor of order one. Interestingly, assuming that the particle behaves as a perfectly polarizable $(K=1)$ sphere with a radius $a_{\text {eff }}$, one can express $\alpha=\alpha_{0}\left(1+\frac{\beta \lambda_{D}}{a}\right)^{3}$, where $a_{0}$ is the polarizability of a perfectly polarizable particle with a radius equal to $a$. Fitting this expression to the data in Figure $4 \mathrm{C}$ reveals that $\beta=2.1 \pm 0.1$ produces good agreement between this model and experiment. Importantly, this calculation provides a simple way to predict the polarizability of an arbitrary particle in a solution with known $\lambda_{D}$. It is important to note that this calculation does not depend on the dielectric properties of the particle, as these are screened by the electric double layer.

An important implication of Figure $4 \mathrm{C}$ is that particles that are small compared to the Debye screening length will appear substantially larger from a DEP perspective. This will likely make nanoparticles significantly easier to manipulate using nanoscale electric fields. This effect has likely remained difficult to explore due to biological samples requiring relatively high salt concentrations to maintain stability while the vast majority of studies observing DEP of particles focus on those that are $100 \mathrm{~nm}$ in size or larger, thus being larger than the Debye screening length in most buffers. The measurement strategy described herein could be used to explore further details of this effect.

Taken together, we have developed a highly quantitative approach to measure $a$ of nanoscale objects at low and intermediate media conductivities. By studying QDs, which are both bright and can be synthesized with a high degree of structural control, we interrogate a regime of $a / \lambda_{D}$ that has been previously inaccessible to quantitative measurements of $a$. Indeed, these measurements produce the striking result that particles exhibit DEP effects that are $\sim 30$ times larger than would be expected using the standard MW formulation for $a$. Not only does this make nanoparticles appealing candidates for control using DEP, but it also contributes to explaining observations of conductivity-dependent DEP of biomolecules. This 
increase in polarizability is consistent with the particles behaving as spheres with a radius that is increased by 2.1 times the Debye length, which itself is a useful relationship for predicting the polarizability of nanoparticles in electrolytic solutions. Looking forward, the polarizability assay developed herein provides new avenues for studying nanoscale constructs to both determine their properties as well as uncover more general relationships regarding the behavior of polarizable objects in electrolytic solutions. Due to the widereaching importance and utility of polarizability, these lessons can be applied to fields ranging from nanomedicine and nanoparticle assembly.

\section{Supplementary Material}

Refer to Web version on PubMed Central for supplementary material.

\section{ACKNOWLEDGMENT}

K.A.B. and W. C. acknowledge support from the Gordon and Betty Moore Foundation and from the American Chemical Society Petroleum Research Fund through award 57452-DNI9. A.M.D received support from the National Center for Advancing Translational Sciences, National Institutes of Health, through BU-CTSI Grant Number 1KL2TR001411. Financial support for M.C. was provided in part through the Clare Boothe Luce (CBL) Program from the Henry Luce Foundation. All authors acknowledge support from the Boston University Photonics Center and membership in the BU Nanotechnology Innovation Center (BUnano). This work was performed in part at the Center for Nanoscale Systems (CNS), a member of the National Nanotechnology Infrastructure Network (NNIN), which is supported by the National Science Foundation under NSF award no. ECS-0335765.

\section{REFERENCES}

1. Modarres P; Tabrizian M Sens. Actuators B 2017, 2017, 391-408.

2. Castellanos A; Ramos A; González A; Green NG; Morgan H J. Phys. D: Appl Phys 2003, 2003, 2584-2597.

3. Demircan Y; Özgür E; Külah H Electrophoresis 2013, 2013, (7), 1008-1027.

4. Verschure R; Ijlst L Nature 1966, 1966, (5049), 619.

5. Brown KA; Westervelt RM Nano Lett. 2011, 2011, (8), 3197-3201.

6. Watarai H; Sakamoto T; Tsukahara S Langmuir 1997, 1997, (8), 2417-2420.

7. Voldman J; Braff RA; Toner M; Gray ML; Schmidt MA Biophys. J. 2001, 2001, 531-541.

8. Fatoyinbo HO; Hoettges KF; Hughes MP Electrophoresis 2008, 2008, (1), 3-10.

9. Vahey MD; Voldman J Anal. Chem 2009, 2009, (9), 10.

10. Sancho M; Martinez G; Munoz S; Sebastian JL; Pethig R Biomicrofluidics 2010, 2010, (2), 022802-11.

11. Weiss NG; Jones PV; Mahanti P; Chen KP; Taylor TJ; Hayes MA Electrophoresis 2011, 2011, (17), 2292-2297.

12. Honegger T; Peyrade D Biomicrofluidics 2012, 2012, (4), 044115.

13. García-Sánchez; Ren Y; Arcenegui JJ; Morgan H; Ramos A Langmuir 2012, 2012, (39), 1386113870.

14. Bakewell DJ; Bailey J; Holmes D Electrophoresis 2015, 2015, (13), 1443-1450.

15. Generalov KV; Generalov VM; Kruchinina MV; Shuvalov GV; Buryak GA; Safatov AS Measurement Techniques 2017, 2017, (1), 82-86.

16. Isaacoff BP; Brown KA Nano Lett. 2017, 2017, (11), 6508-6510.

17. Zhu J; Ozdemir SK; Xiao Y-F; Li L; He L; Chen D-R; Yang L Nat. Photonics 2010, 2010, (1), 46.

18. Chrimes AF; Kayani AA; Khoshmanesh K; Stoddart PR; Mulvaney P; Mitchell A; Kalantar-Zadeh K Lab Chip 2011, 2011, (5), 921-928.

19. Zhu J; Özdemir ŞK; He L; Chen D-R; Yang L Optics express 2011, 2011, (17), 16195-16206. 
20. Sin MLY; Gau V; Liao JC; Haake DA; Wong PK J. Phys. Chem. C 2009, 2009, (16), 6561-6565.

21. Zhou H; Preston MA; Tilton RD; White LR J. Colloid Interface Sci. 2005, 2005, (2), 845-856.

22. Zhao K; He K Phys. Rev. B 2006, 2006, (20), 205319.

23. Shilov V Colloid Journal 2008, 2008, (4), 515-528.

24. Zhao H; Bau HH J. Colloid Interface Sci. 2009, 2009, (2), 663-671.

25. Zhao H; Bau HH Langmuir 2009, 2009, (8), 5412-5420.

26. Mangelsdorf CS; White LR J. Chem. Soc. Faraday Trans. 1997, 1997, (17), 3145-3154.

27. Hill RJ; Saville D; Russel W Phys. Chem. Chem. Phys. 2003, 2003, (5), 911-915.

28. Hill RJ Proc R Soc Lond A Math Phys Sci 2015, 2015, (2183), 20150522.

29. Hollingsworth A; Saville DJ Colloid Interface Sci. 2004, 2004, (1), 235-245.

30. Jiménez ML; Delgado A; Ahualli S; Hoffmann M; Witteman A; Ballauff M Soft Matter 2011, 2011, (8), 3758-3762.

31. Beltramo PJ; Roa R; Carrique F; Furst EM J. Colloid Interface Sci. 2013, 2013, 54-58.

32. Hill RJ Soft matter 2016, 2016, (38), 8030-8048.

33. Giraud G; Pethig R; Schulze H; Henihan G; Terry JG; Menachery A; Ciani I; Corrigan D; Campbell CJ; Mount AR Biomicrofluidics 2011, 2011, (2), 024116.

34. Stellwagen NC Biopolymers 1981, 1981, (3), 399-434.

35. Suzuki S; Yamanashi T; Tazawa S.-i.; Kurosawa O; Washizu M IEEE Trans. Ind Appl 1998, 1998, (1), 75-83.

36. Bakewell D; Ermolina I; Morgan H; Milner J; Feldman Y Biochim. Biophys. Acta, Gene Struct. Expression 2000, 2000, (1-2), 151-158.

37. Regtmeier J; Duong TT; Eichhorn R; Anselmetti D; Ros A Anal. Chem 2007, 2007, (10), 39253932.

38. Tuukkanen S; Kuzyk A; Toppari JJ; Häkkinen H; Hytönen VP; Niskanen E; Rinkiö M; Törmä P Nanotechnology 2007, 2007, (29), 295204.

39. Lapizco-Encinas BH; Ozuna-Chacón S; Rito-Palomares MJ Chromatogr. A 2008, 2008, (1), 4551.

40. Henning A; Henkel J; Bier FF; Hölzel R PMC Biophys. 2008, 2008, (1), 4.

41. Parikesit GO; Markesteijn AP; Piciu OM; Bossche A; Westerweel J; Young IT; Garini Y Biomicrofluidics 2008, 2008, (2), 024103.

42. Regtmeier J; Eichhorn R; Bogunovic L; Ros A; Anselmetti D Anal. Chem. 2010, 2010, (17), 7141-7149.

43. Henning A; Bier FF; Hölzel R Biomicrofluidics 2010, 2010, (2), 022803.

44. Nakano A; Chao TC; Camacho-Alanis F; Ros A Electrophoresis 2011, 2011, (17), 2314-2322.

45. Martinez-Duarte R; Camacho-Alanis F; Renaud P; Ros A Electrophoresis 2013, 2013, (7), 11131122.

46. Camacho-Alanis F; Ros A Bioanalysis 2015, 2015, (3), 353-371.

47. Swami N; Chou C-F; Ramamurthy V; Chaurey V Lab Chip 2009, 2009, (22), 3212-3220.

48. Barrett LM; Skulan AJ; Singh AK; Cummings EB; Fiechtner GJ Anal. Chem. 2005, 2005, (21), 6798-6804.

49. Zhao H Electrophoresis 2011, 2011, (17), 2232-2244.

50. Chen Y; Vela J; Htoon H; Casson JL; Werder DJ; Bussian DA; Klimov VI; Hollingsworth JA J. Am. Chem. Soc. 2008, 2008, (15), 5026-5027.

51. Chern M; Nguyen TT; Mahler AH; Dennis AM Nanoscale 2017, 2017, (42), 16446-16458.

52. Ghosh Y; Mangum BD; Casson JL; Williams DJ; Htoon H; Hollingsworth JA J. Am. Chem. Soc. 2012, 2012, (23), 9634-9643.

53. Nasilowski M; Spinicelli P; Patriarche G; Dubertret B Nano Lett. 2015, 2015, (6), 3953-3958.

54. Di Corato R; Quarta A; Piacenza P; Ragusa A; Figuerola A; Buonsanti R; Cingolani R; Manna L; Pellegrino T J. Mater. Chem 2008, 2008, (17), 1991-1996.

55. Medintz IL; Uyeda HT; Goldman ER; Mattoussi H Nature Mater. 2005, 2005.

56. Lakowicz JR, Principles of fluorescence spectroscopy. Springer Science \& Business Media: 2013. 
57. Pons T; Uyeda HT; Medintz IL; Mattoussi HJ Phys. Chem. B 2006, 2006, (41), 20308-20316.

58. Wittgren B; Wahlund K-G; Dérand H; Wesslén B Langmuir 1996, 1996, (25), 5999-6005.

59. Min GK; Bevan MA; Prieve DC; Patterson GD Colloids Surf. A Physicochem. Eng. Asp 2002, 2002, (1), 9-21. 

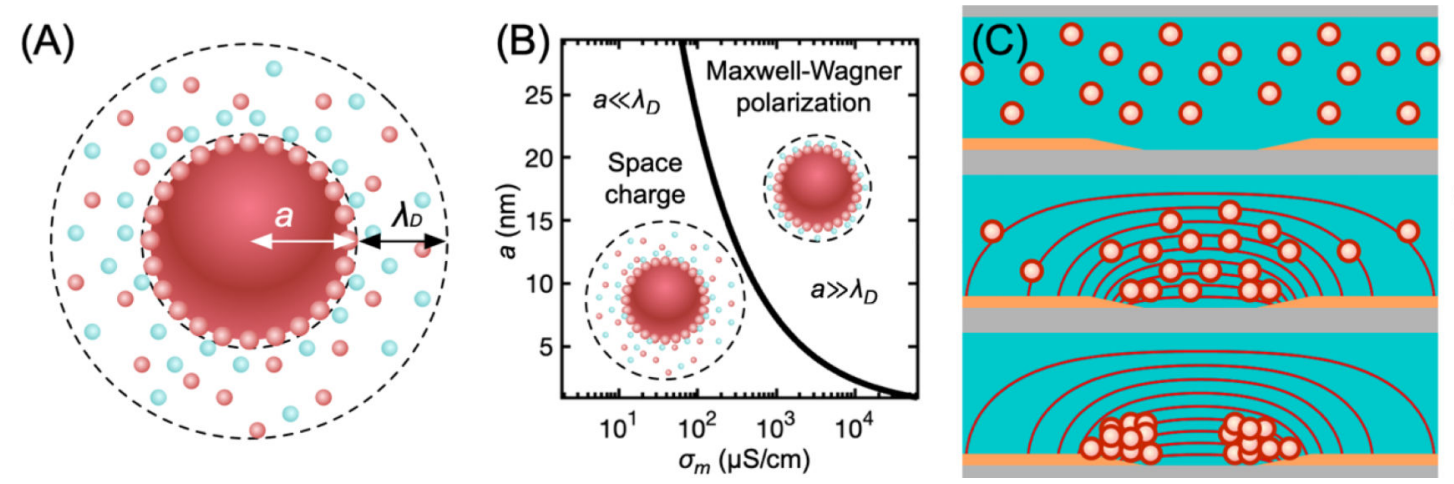

Brownian
$\frac{1}{2} \alpha E^{2} \ll k_{B} T$
Concentration
enhancement
$\frac{1}{2} \alpha E^{2} \sim k_{B} T$
Trapping
$\frac{1}{2} \alpha E^{2} \gg k_{B} T$

Figure 1.

(A) A nanoparticle in an aqueous solution is defined by its radius $a$ and the Debye length $\lambda_{D}$. Positively and negatively charged ions (blue and red spheres) participate in electrostatic screening of the particle. (B) The nanoparticle polarizability $a$ is expected to exhibit two regimes of behavior depending on the medium conductivity $\sigma_{m}$ and corresponding $\lambda_{D}$ relative to a. (C) Dielectrophoresis (DEP) exhibits different categories of behavior depending on the relative importance of thermal energy defined by the Boltzmann constant $k_{B}$ and absolute temperature $T$ and the energy associated with the induced dipole in an electric field $E$. 

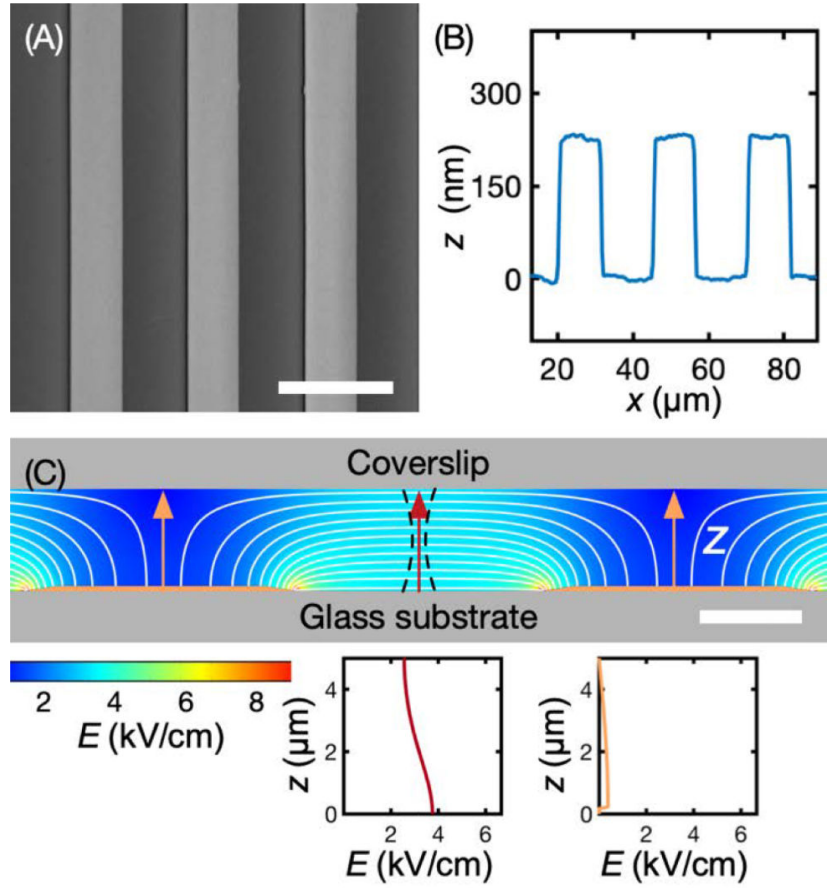

(D)

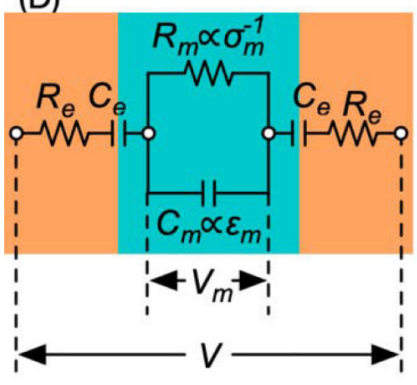

(E)

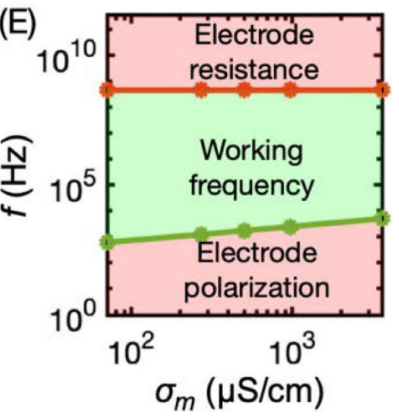

Figure 2.

(A) Scanning electron micrograph of a set of indium tin oxide (ITO) electrodes. Scale bar is $25 \mu \mathrm{m}$. (B) Atomic force microscope height trace showing the profile of the electrode array. (C) Finite element electric field calculation of the field in the gap region with $5 \mathrm{~V}$ applied between the electrodes. Scale bar is $5 \mu \mathrm{m}$. The insets are the magnitude of electric field $E$ vs. distance $z$ from the electrodes in the high field gap region (red) or the low field region above the electrode (orange). The point spread function of the optical microscope is shown as a dashed line for reference. (D) Lumped element model of the testing circuit including the electrode resistance $R_{e}$ electrode-solution capacitance $C_{e}$, medium resistance $R_{m}$, and medium capacitance $C_{m}$, which is proportional to its dielectric permittivity $\varepsilon_{m}$. (E) The working frequency is defined as the range in $f$ in which the voltage on the electrodes $V_{m}$ is attenuated by less than $1 \mathrm{~dB}$ relative to the applied voltage $V$, as calculated by the lumped element circuit model. Descriptions of how the lumped element parameters are calculated and how these are used to define a working frequency are given in the SI. 

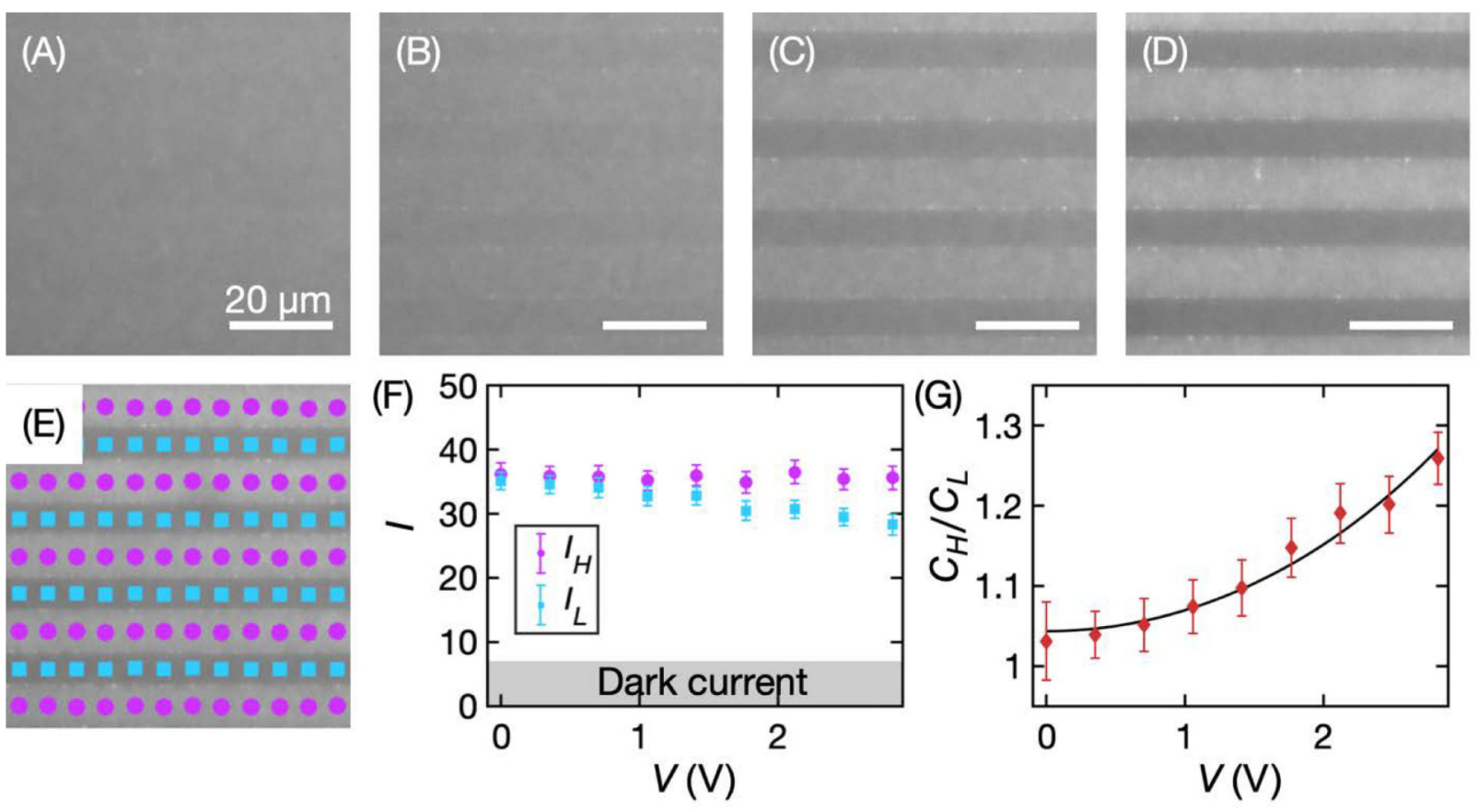

Figure 3.

Fluorescent micrographs of a fluid cell filled with $25 \mathrm{nM}$ QDs and applied root-meansquared voltage (A) $V=0 \mathrm{~V}$, (B) $V=0.71 \mathrm{~V}$, (C) $V=1.76 \mathrm{~V}$, and (D) $V=2.82 \mathrm{~V}$. (E) Graphical representation of how the image processing script chose high (purple circles) and low (blue squares) field regions. (F) Camera intensity $I$ recorded in the high field $\left(I_{H}\right)$ and low field $\left(I_{L}\right)$ regions. (G) Ratio of concentration in high field region $C_{H}$ to concentration in low field region $C_{L}$ with the black line showing a fit to Equation (5). 

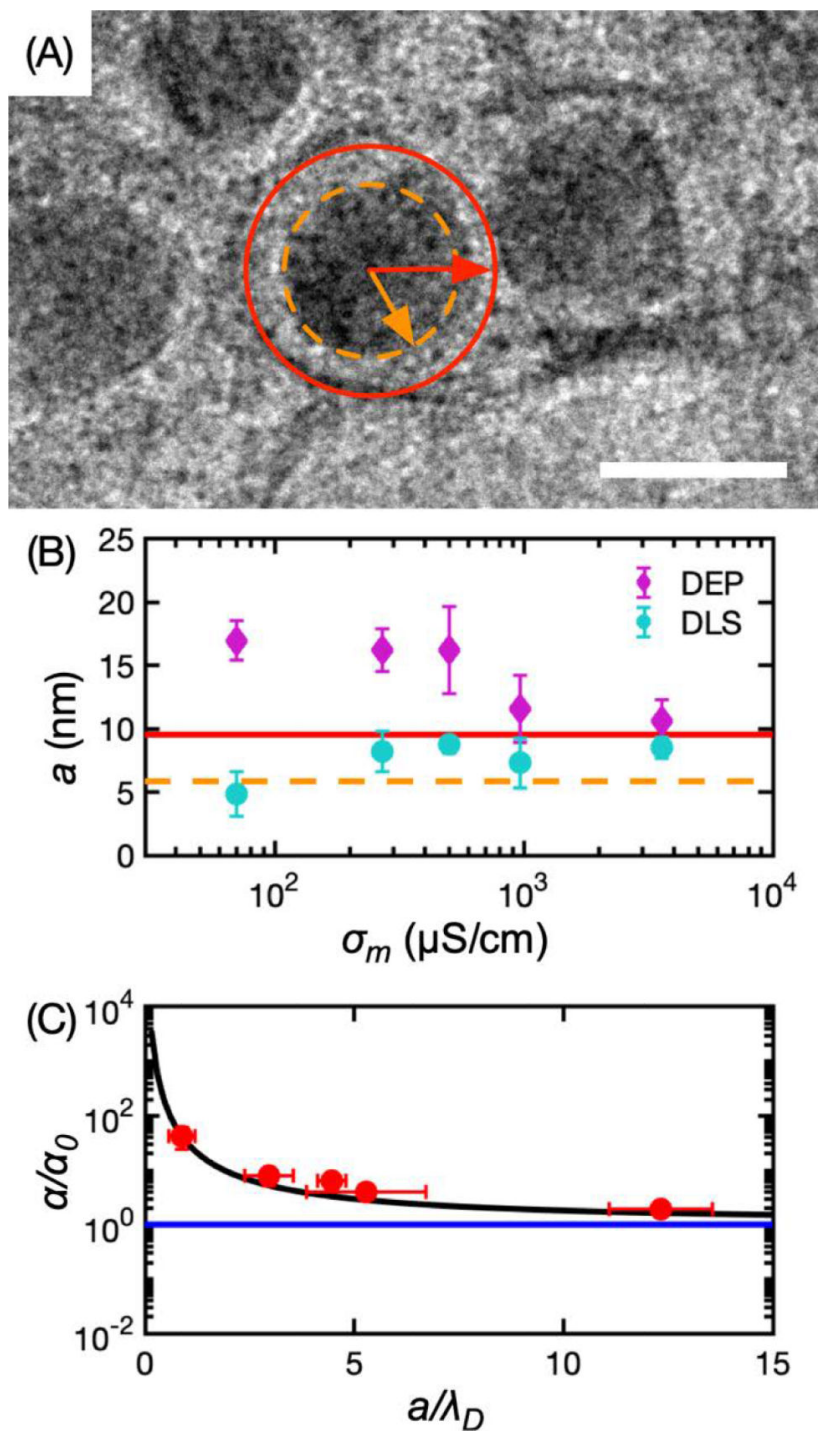

Figure 4.

(A) Transmission electron micrograph (TEM) showing a negative stain of QDs with the inorganic core radius (orange) and ligand shell radius (red). Scale bar is $15 \mathrm{~nm}$. (B)

Apparent a from dynamic light scattering (DLS) and from the DEP measurements compared with average values from TEM (horizontal lines). Agreement amongst all three occurs at high $\sigma_{m}$. (C) Ratio of measured $a$ to expected polarizability $a_{0}$ of a sphere with a computed for various values of $a / \lambda_{D}$. using values of $a$ from DLS. The lines represent the expected asymptotic value (blue) and a fit to an effective sphere model in which $a_{e f f}=a+\beta \lambda_{D}$ (black). 